\title{
A TEORIA DAS REPRESENTAÇÕES SOCIAIS NAS DISSERTAÇÕES E TESES EM ENFERMAGEM: UM PERFIL BIBLIOMÉTRICO
}

\author{
Ana Maria Farias da Silva', Jussara Gue Martini², Sandra Greice Becker ${ }^{3}$
}

\footnotetext{
${ }^{1}$ Doutoranda do Programa de Pós-Graduação em Enfermagem (PEN) da Universidade Federal de Santa Catarina (UFSC). Professora Assistente do Departamento de Enfermagem da UFSC. Santa Catariana, Brasil. E-mail: anamaria@nfr.ufsc.br

${ }^{2}$ Doutora em Educação. Professora do Departamento de Enfermagem e do PEN/UFSC. Santa Catariana, Brasil. E-mail: jussarague@gmail.com

${ }^{3}$ Doutoranda do PEN/UFSC. Docente na Escola de Enfermagem da Universidade Federal do Amazonas. Bolsista Fundação de Amparo à Pesquisa do Amazonas. E-mail: sgbecker@ufam.edu.br
}

RESUMO: A investigação buscou identificar dissertações e teses de enfermagem, disponíveis na base de dados do Centro de Estudos e Pesquisas em Enfermagem que utilizaram a Teoria das Representações Sociais, no período de 2001 a 2007. Trata-se de estudo exploratório, com análise bibliométrica dos trabalhos registrados na base. Das 3246 unidades encontradas, 167 (5\%) utilizaram a teoria, sendo 38 (22,7\%) teses e 129 (77,3\%) dissertações. A região Sudeste, com 61,2\%, e a Nordeste, com 26,8\% registram a maior produção. Quanto à abordagem da teoria, em $80(62 \%)$ das dissertações e em 27 (71\%) das teses ela foi o referencial; seu uso como referencial teórico-metodológico ocorreu em 16 (10\%) estudos. Sobre os instrumentos de coleta de dados, a entrevista foi utilizada em $44,4 \%$ dos casos; e a análise de conteúdo foi opção em $54,6 \%$ dos estudantes. Destaca-se o crescente interesse pela teoria e a necessidade de maior critério na elaboração dos resumos, considerando sua importância na divulgação da produção científica.

DESCRITORES: Pesquisa em enfermagem. Psicologia social. Enfermagem. Bibliometria.

\section{THE SOCIAL REPRESENTATION THEORY IN GRADUATE NURSING DISSERTATIONS AND THESES: A BIBLIOMETRIC PROFILE}

\begin{abstract}
This study aimed to identify nursing theses and dissertations which utilized the Social Representation Theory available in the Center for Nursing Studies and Research database from 2001 to 2007. It is an exploratory, Bibliometrics analysis study of the papers registered in the database. Of the 3246 studies found, 167 (5\%) used the Theory; 38 (22.7\%) were dissertations and 129 (77.3\%) were theses. The Southeast and the Northeast regions recorded the highest production, with $61.2 \%$ and $26.8 \%$, respectively. In theoretical approach, $80(62 \%)$ theses and $27(71 \%)$ dissertations used this theoretical framework. Its use as a theoretical and methodological reference occurred in $16(10 \%)$ studies. In these studies data was collected through interviews, used in $44.4 \%$ of the cases, and content analysis, the choice of $54.6 \%$ of the students. We highlight a growing interest in the Social Representation Theory and the need for greater criteria in preparing abstracts, given their importance in developing scientific research.
\end{abstract}

DESCRIPTORS: Research nursing. Psychology social. Nursing. Bibliometrics

\section{LA TEORÍA DE LAS REPRESENTACIONES SOCIALES EN LAS TESINAS Y TESIS DE ENFERMERÍA: UN PERFIL BIBLIOMÉTRICO}

RESUMEN: La investigación tuvo como objetivo identificar las tesinas y tesis de enfermería disponibles en la base de datos del Centro de Estudios e Investigación en Enfermería que han empleado la Teoría de las Representaciones Sociales, en el período de 2001 a 2007. Se trata de un estudio exploratorio, con análisis bibliométrico de los trabajos registrados en esa base de datos. De las 3.246 unidades encontradas, $167(5 \%)$ utilizaron esa teoría, 38 (22,7\%) son tesis y $129(77,3 \%)$ tesinas. La región Sudeste $(61,2 \%)$ y la Nordeste $(26,8 \%)$ registraron la mayor producción. En relación a la teoría, en $80(62 \%)$ tesinas y en 27 (71\%) tesis se empleó como referencial teórico; su uso como referencial teórico-metodológico está presente en $16(10 \%)$ estudios. Sobre los instrumentos de recolección de datos, la entrevista fue utilizada en el 44,4\% de los casos; el análisis de contenido fue la opción elegida (54,6\%) por los estudiantes. Se destaca el creciente interés por la Teoría de las Representaciones Sociales y la necesidad de un mayor rigor en la elaboración de los resúmenes, considerando su importancia para la divulgación de la producción científica.

DESCRIPTORES: Investigación en enfermería. Psicología social. Enfermería. Bibliometría 


\section{INTRODUÇÃO}

A Teoria das Representações Sociais ${ }^{1}$ difundiu-se no Brasil a partir dos anos 80, com o surgimento dos primeiros estudos de mestrado e doutorado. Tem como primeiro autor Serge Moscovici e, como reconhecida colaboradora e difusora da teoria, tanto na Europa, como no Brasil, Denise Jodelet. ${ }^{2}$ Como uma teoria de vitalidade, muitos estudiosos têm contribuído na discussão dos aspectos teóricos e metodológicos dentro do campo das representações sociais..$^{3-10}$

Na década de 90, houve a criação das Jornadas Internacionais sobre Representações Sociais, sendo que a primeira ocorreu na cidade de Natal, em 1998 e, desde então, vem ocorrendo bianualmente. Estes são momentos de troca, tanto em relação ao conhecimento e atualização sobre a teoria, como em relação aos aspectos metodológicos que envolvem as pesquisas que focalizam este tipo de fenômeno. ${ }^{10}$

A teoria, nas últimas décadas, tem sido empregada em vários campos do conhecimento, além da psicologia social, como na educação, comunicação, tecnologia, saúde (saúde coletiva, enfermagem, medicina, odontologia), entre outros. Na área da saúde, é destacada por abordar os fenômenos de natureza sócio-cultural, relativos à vida social dos grupos, expressa por valores, noções e regras sociais que regem as relações dos sujeitos com o mundo e com os outros, orientando e organizando as suas condutas/comportamentos e as comunicações sociais. ${ }^{11}$

Podemos identificar inúmeras publicações que retratam a importância das representações sociais nas diferentes possibilidades de intervenções em saúde. Um levantamento da produção brasileira no campo das representações sociais, no período de 1988 a 1997, reuniu em torno de 867 títulos. Nessa oportunidade verificou-se que cerca de $28 \%$ desse conjunto era relativo a estudos da área da saúde. ${ }^{12} \mathrm{~A}$ relevância da teoria no campo da enfermagem, em particular, oferece suporte teórico aos pesquisadores, possibilitando compreender o modo como os indivíduos cuidam da sua saúde, suas atitudes, valores, e significados frente ao adoecimento e a terapêutica. Outro aspecto que determina o desenvolvimento de estudos sobre representação social, no âmbito da saúde, é o desejo de compreender o processo saúde-doença para além do modelo biomédico, cujo enfoque central é a biologia humana, a doença e a fisiopatologia.

Na Enfermagem, o paradigma das representações sociais tem despertado crescente interesse.
No Rio de Janeiro, a psicóloga Ângela Arruda, na década de 1990, foi quem trouxe a teoria para o Brasil e a introduziu, inicialmente, no Mestrado da Escola de Enfermagem Anna Nery. ${ }^{6}$

As pesquisas podem trazer subsídios para o fortalecimento de um novo modelo de atenção à saúde. Nesse sentido, as representações sociais devem ser estudadas articulando-se elementos afetivos, mentais e sociais e integrando ao lado da cognição, da linguagem e da comunicação, a consideração das relações sociais que afetam as representações e a realidade material, social e ideativa sobre a qual elas têm de intervir. ${ }^{2}$

Sobre as representações sociais, pode-se dizer que elas circulam, cruzam-se e se concretizam incessantemente através de uma fala, um gesto, um encontro, em nosso universo cotidiano. ${ }^{1}$ Quando se pesquisa representações sociais, estuda-se seres humanos que pensam de forma autônoma, produzem e comunicam representações constantemente. ${ }^{1}$

A teoria das representações tem sido empregada em dissertações e teses nos programas de pós-graduação, com muitas discussões e avanços sobre suas vertentes teóricas e o aprimoramento metodológico. Então, buscou-se, nessa investigação, responder à seguinte questão norteadora: qual foi a utilização da Teoria das Representações Sociais na produção científica da enfermagem?

Nessa perspectiva, este estudo teve como objetivo identificar a produção científica publicada em dissertações e teses na área de enfermagem à luz da Teoria das Representações Sociais.

Assim sendo, o interesse em conhecer a produção científica sobre as representações sociais nos estudos de pós-graduação stricto-sensu, no Brasil, motivou a presente pesquisa.

\section{METODOLOGIA}

Trata-se de um estudo exploratório, com abordagem bibliométrica, realizado mediante a análise dos resumos de dissertações e teses registradas na base de dados do Centro de Estudo de Pesquisa em Enfermagem (CEPEn), disponível on-line ou em forma de CD-ROM, no período de 2001 a 2007.13 Esse banco de dados dispõe de informações sobre Pesquisa e Pesquisadores de Enfermagem, considerado o maior banco de teses e dissertações na área de Enfermagem do Brasil, organizado pela Associação Brasileira de Enfermagem (ABEn), foi criado 
em 17 de julho de 1971, destinado a incentivar o desenvolvimento e a divulgação da pesquisa em enfermagem, organizar e preservar documentos históricos da profissão.

As pesquisas bibliométricas se apresentam como um importante método para analisar e avaliar os processos relacionados à atividade científica e, a partir da aplicação de um conjunto de técnicas, quantificar o processo de comunicação científica armazenada em fontes de informação. ${ }^{14}$ Além disso, apóia-se em estudos realizados em bases de dados bibliográficos, indexadores e resumos, em diretórios e catálogos de títulos de periódicos e em referências e citações. ${ }^{15}$

Utilizou-se como critério de inclusão todos os resumos de teses e dissertações disponíveis no banco de dados, que mencionassem as expressões "representações sociais" ou "representação social". O período de coleta foi de março a junho de 2009, sendo que, na data da coleta estavam disponíveis on-line os dados catalogados de 2001 a 2007.

Após a identificação dos estudos, foi feita a leitura analítica dos resumos utilizando um roteiro, considerando às seguintes variáveis: tipo de publicação, área temática, ano de defesa, tipo de instituição de ensino, região do país. Quanto às questões relacionadas com o referencial metodológico, foram investigados o grupo de amostragem, coleta e análise dos dados, bem como, o emprego do referencial teórico. Os dados extraídos foram registrados e organizados em um instrumento no formato de tabela, construído a partir das variáveis estabelecidas.

Para análise dos dados foi utilizado o método descritivo por variáveis, sendo a discussão realizada com autores cuja produção é pertinente para analise do objeto deste estudo. A apresentação dos resultados considerou a estatística descritiva simples: freqüência absoluta e percentual.

Como cuidados éticos, ainda que os dados sejam de domínio público, foi solicitada autorização de pesquisa para a responsável pelo Banco de Dados de Teses e Dissertações do CEPEn e as autorias das obras foram tratadas no seu conjunto, evitando assim qualquer citação ou identificação de forma particular.

\section{RESULTADOS E DISCUSSÃO}

Conforme os dados do CEPEn, nos catálogos de 2001 a 2007,13 pode-se constatar 3.246 trabalhos registrados incluindo 832 teses e 2.414 dissertações. Destes, 167 pesquisas utilizaram a teoria das representações sociais, sendo 129 dissertações e 38 teses, o que representa um percentual de $5 \%$ dos estudos conforme apresentado no tabela 1.

\section{Tabela 1 - Distribuição dos estudos embasados na Teoria das Representações Sociais}

\begin{tabular}{lrrrrrrrrr}
\hline \multirow{2}{*}{ Tipo de estudo } & \multicolumn{10}{c}{ Anos } \\
\cline { 2 - 12 } & $\mathbf{2 0 0 1}$ & $\mathbf{2 0 0 2}$ & $\mathbf{2 0 0 3}$ & $\mathbf{2 0 0 4}$ & $\mathbf{2 0 0 5}$ & $\mathbf{2 0 0 6}$ & $\mathbf{2 0 0 7}$ & $\mathbf{f}$ & $\%$ \\
\hline Tese & 7 & 8 & 4 & 5 & 10 & 2 & 2 & 38 & 22,7 \\
Dissertação & 25 & 21 & 13 & 12 & 23 & 26 & 9 & 129 & 77,3 \\
\hline Total & $\mathbf{3 2}$ & $\mathbf{2 9}$ & $\mathbf{1 7}$ & $\mathbf{1 7}$ & $\mathbf{3 3}$ & $\mathbf{2 8}$ & $\mathbf{1 1}$ & $\mathbf{1 6 7}$ & $\mathbf{1 0 0}$ \\
\hline
\end{tabular}

Com relação à frequência das teses e dissertações, percebe-se a predominância de ocorrência no ano de 2005, que somam 33(19,7\%). Já o menor índice foi em 2007, com 11 (6,5\%) estudos. Considerando a produção de dissertações, estas totalizam $129(77,3 \%)$, enquanto que as teses representam $38(22,7 \%)$ dos estudos com representações sociais.

O crescimento da produção acadêmica é resultante da consolidação da teoria das representações sociais no Brasil, evidente por várias modalidades de produção científica, desde a publicação de livros, artigos em periódicos, apresentação de estudos em eventos científicos, até a realização da Jornada Internacional de Representações Socais, por diversas vezes, neste país. Outro dado, é a expansão dos Programas e cursos de Pós-Graduação stricto sensu, na área de Enfermagem, que somam atualmente 50 cursos, destes 22 de mestrado e doutorado, 19 de mestrado, sete de mestrado profissional e dois de doutorado, reconhecidos pela Coordenação de Aperfeiçoamento de Nível Superior (CAPES). ${ }^{16}$ Considera-se ainda que no Brasil o tempo médio de duração de um curso de mestrado é de dois anos e, de doutorado, quatro anos. 
Tabela 2 - Distribuição dos estudos segundo o ano e a instituição de ensino

\begin{tabular}{|c|c|c|c|c|c|c|c|c|c|}
\hline \multirow{2}{*}{ Instituições de ensino } & \multicolumn{7}{|c|}{ Anos } & \multirow[b]{2}{*}{ f } & \multirow[b]{2}{*}{$\%$} \\
\hline & 2001 & 2002 & 2003 & 2004 & 2005 & 2006 & 2007 & & \\
\hline Universidade do Rio de Janeiro & 6 & 4 & 3 & 2 & 1 & 5 & 2 & 23 & 13,8 \\
\hline Universidade Federal do Rio de Janeiro & 6 & 4 & 4 & 4 & 4 & 1 & - & 23 & 13,8 \\
\hline Universidade de São Paulo (USP) & 5 & 7 & 3 & 1 & 3 & 1 & 1 & 21 & 12,6 \\
\hline USP Ribeirão Preto & 5 & 2 & - & - & 3 & 1 & - & 11 & 6,6 \\
\hline Universidade Estadual de Campinas & - & 1 & 1 & - & 5 & 1 & 1 & 9 & 5,4 \\
\hline Universidade Federal da Bahia & 3 & 5 & 1 & 2 & 8 & 6 & 2 & 27 & 16,0 \\
\hline Universidade Federal do Ceará & 1 & 2 & 2 & 2 & 2 & - & - & 9 & 5,4 \\
\hline Universidade Federal de Santa Catarina & - & 2 & - & 1 & 1 & 3 & 2 & 9 & 5,4 \\
\hline Universidade Federal da Paraíba & 3 & - & 2 & - & - & 1 & 1 & 7 & 4,2 \\
\hline Universidade de Minas Gerais & 2 & 1 & 1 & 5 & 1 & 4 & 1 & 15 & 9,0 \\
\hline Universidade Federal do Paraná & - & - & - & - & 1 & 2 & 1 & 4 & 2,4 \\
\hline Universidade Federal de Goiás & - & 1 & - & - & 2 & - & - & 3 & 1,8 \\
\hline Universidade de Brasília & 1 & - & - & - & - & 1 & - & 2 & 1,2 \\
\hline Universidade Estadual de Maringá & - & - & - & - & - & 2 & - & 2 & 1,2 \\
\hline Universidade Rio Grande Norte & - & - & - & - & 2 & - & - & 2 & 1,2 \\
\hline Total & 32 & 29 & 17 & 17 & 33 & 28 & 11 & 167 & 100,0 \\
\hline
\end{tabular}

Nota-se na tabela 2, que a Universidade Federal da Bahia apresenta o maior número de dissertações, ou seja, 27 (16\%), seguido da Universidade do Rio de Janeiro (UNIRIO - Escola Enfermagem Alfredo Pinto), com 23 (13,8\%) dissertações, sendo que ambas não possuem teses. A Universidade Federal do Rio de Janeiro (Escola de Enfermagem Anna Nery) registra 10 teses e 13 dissertações, totalizando 23 (13,8\%); a Universidade de São Paulo, 10 teses e 11 dissertações, que representa 21 $(12,6 \%)$; a Universidade Federal de Minas Gerais, com 15 dissertações $(9,0 \%)$ e a Universidade de São Paulo - Escola de Enfermagem de Ribeirão Preto com nove teses e duas dissertações num total de 11 (6,6\%). A menor incidência é apontada nas Universidades de Brasília, Estadual de Maringá e Universidade do Rio Grande do Norte, com duas dissertações $(1,2 \%)$.

Percebe-se a concentração de pesquisas sobre representações sociais na região Sudeste $102(61,2 \%)$, representado por seis instituições, localizadas principalmente nos estados de São Paulo e Rio de Janeiro. A região Nordeste, com $43(26,8 \%)$, compreendendo os Estados da Bahia, Paraíba, do Ceará e Rio Grande do Norte. Já em menor porcentagem, encontra-se a região Sul, com $15(9,0 \%)$ e, por último a região Centro-Oeste, com cinco $(3,0 \%)$. Evidencia-se a ausência de estudos realizados na região Norte, fator que pode estar relacionado à ausência de cursos de pós-graduação stricto sensu na área da enfermagem.

Vale ressaltar que esses dados apresentam similaridade com o mapa de localização dos Progra- mas de Pós-Graduação de mestrado e doutorado em enfermagem no Brasil reconhecido pelo Ministério da Educação, sendo que 19(48,8\%) situam-se no Sudeste, seguido de nove $(23 \%)$ no Nordeste, oito $(20,5 \%)$ no Sul, três $(7,7 \%)$ no Centro-Oeste e nenhum programa é citado na região Norte. ${ }^{16}$

Constata-se, ainda, que, das 167 dissertações e teses analisadas, $129(77 \%)$ são oriundas de Instituições de Ensino Superior (IES), públicas federais, e 38 (23\%), de IES públicas estaduais e nenhuma em instituição de ensino particular. Chama a atenção a importante representatividade das instituições públicas na formação de mestres e doutores, contribuindo para o fortalecimento da pós-graduação e da produção científica no país. Seu papel é social e constitui-se como centro criador da ciência e cultura, construindo o alicerce para o desenvolvimento da pesquisa. ${ }^{17}$

Nos estudos analisados, foram categorizadas sete áreas temáticas, sendo a de maior ocorrência, o processo de trabalho e educação em enfermagem e saúde, com $42(25,1 \%)$, seguido da saúde do adulto com $39(23,3 \%)$, saúde da mulher com 30 (18\%), saúde da criança e adolescente, $19(11,3 \%)$, saúde do idoso, $15(9 \%)$, saúde mental, $14(8,3 \%)$ e o tema de menor incidência foi a saúde coletiva, com oito (5\%).

A predominância das temáticas pode estar relacionada às linhas de pesquisas descritas nos Programas de Pós-Graduação, assim como a adoção de políticas publicas nos serviços de saúde voltados ao atendimento da área de saúde da mulher, da criança, e adultos com doenças crônicas. A 
baixa incidência do tema saúde mental e do idoso revela ainda o grande desafio social e político de articulação no sistema de saúde, a fim de atender os múltiplos aspectos do processo saúde-doença dessa população.

Esses achados evidenciam o quanto a teoria das representações sociais tem oferecido um importante suporte teórico aos pesquisadores da área de enfermagem, na compreensão dos fenômenos do cotidiano, entre eles a saúde e a doença dos diversos grupos sociais. Apesar da consolidação da teoria das representações sociais no campo do conhecimento em enfermagem, chama a atenção dos pesquisadores e estudiosos a abordagem da referida teoria nos estudos, decorrentes da ambiguidade conceitual e do seu caráter interdisciplinar. ${ }^{11}$

Os estudos examinados adotam a teoria das representações sociais de formas diferenciadas, sendo que $62 \%$ das dissertações e $71 \%$ das teses a utilizam como referencial teórico. Por outro lado, foi considerada como referencial teórico-metodo- lógico em $10 \%$ dos estudos, e como método, em $8,0 \%$ das dissertações e $6,0 \%$ das teses.

Embora a consideração das representações sociais como referencial teórico-metodológico e como método tenha sido minoritária, essa multiplicidade de usos da teoria também foi observada em um estudo sobre os resumos apresentados nas Jornadas Internacionais de Representações Sociais, em que o autor explica que isto indica um equívo$\mathrm{co}$, pois representações sociais não se constituem em uma abordagem metodológica, mas se referem a um fenômeno social e uma teoria científica que se propõe a explicá-lo. ${ }^{10}$

Ainda quanto ao caráter de uso da teoria, este não foi explicitado no resumo em $20 \%$ das dissertações e 13\% das teses. Esse fato pode ser relacionado à ambiguidade do conceito sobre representações sociais, o qual comporta diferentes definições, em contextos teóricos diversos, sendo que, muitas vezes, a teoria é apenas nomeada nas investigações, sem que se evidencie seu emprego real. ${ }^{11}$

\section{Tabela 3 - Instrumento utilizado na coleta de dados}

\begin{tabular}{|c|c|c|c|c|}
\hline Instrumento de coleta de dados & Teses & Dissertação & $f$ & $\%$ \\
\hline Entrevista semi-estruturada & 10 & 64 & 74 & 44,4 \\
\hline Entrevista + Observação & 6 & 12 & 18 & 10,7 \\
\hline Entrevista + Associação livre de palavras & 10 & 26 & 36 & 21,5 \\
\hline Entrevista + Grupo focal & 1 & 3 & 4 & 2,4 \\
\hline Entrevista + DSC* & 1 & 4 & 5 & 3,0 \\
\hline Associação livre de palavras + Grupo focal & 1 & - & 1 & 0,6 \\
\hline Grupo Focal & 2 & 1 & 3 & 1,8 \\
\hline $\mathrm{DSC}^{*}$ & - & 5 & 5 & 3,0 \\
\hline Entrevista não estruturada & - & 2 & 2 & 1,2 \\
\hline Outros & 4 & 1 & 5 & 3,0 \\
\hline Não informa & 2 & 12 & 14 & 8,4 \\
\hline Total & 38 & 129 & 167 & 100 \\
\hline
\end{tabular}

* Dinâmica de Criatividade e Sensibilidade

Em relação aos aspectos metodológicos, a Tabela 3 informa sobre os instrumentos utilizados na coleta de dados. Identificamos que a técnica de entrevistas foi o instrumento de preferência em $74(44,4 \%)$ dos estudos avaliados, seguido da associação livre de palavras, em conjunto com a entrevista, aplicado em 10 teses e 26 dissertações, num total de $36(21,5 \%)$. Foram citados com menor frequência grupo focal, questionário, entrevista não estruturada, dinâmica de sensibilidade e criatividade associada à entrevista, entre outros. Resumos sem indicação do instrumento de coleta foram detectados em 14 pesquisas $(8,4 \%)$.

A entrevista em profundidade nos estudos de representações é defendida por pesquisadores da área, pois ela é capaz de produzir um material discursivo que possibilita evidenciar as representações. Recomenda-se que ela deve começar com perguntas de caráter mais concreto, factuais e relacionadas às experiências cotidianas dos sujeitos e, gradativamente, passar a perguntas que envolvam reflexões mais abstratas e julgamentos. ${ }^{6}$

A associação livre de palavras vem sendo amplamente utilizada nas pesquisas sobre as representações sociais, principalmente naquelas que utilizam a abordagem estrutural, na perspectiva de delineamento do núcleo central.

As formas de coleta de dados empregadas foram, algumas vezes, associadas, o que podemos chamar de multimétodo. A coleta de dados geral- 
mente estabelece um processo de comunicação com o universo estudado, sendo que o recurso de utilizar metodologias combinadas pode ser frutífero por facilitar uma angulação variada do objeto, expondo mais da sua complexidade de encontro com a perspectiva da teoria das representações sociais. ${ }^{18}$

No que tange ao número de sujeitos, aproximadamente $44(34,1 \%)$ das dissertações e 15 $(39,4 \%)$ das teses não apresentaram esta informação; o número mais expressivo se encontra na faixa entre 11 a 30 sujeitos evidenciado em 41 (32,5\%) das dissertações e $10(26,3 \%)$ das teses. O maior número de participantes foi de 300 indivíduos, evidenciado em duas dissertações, e o mínimo foi um estudo com dois participantes.

Algumas vezes, a decisão do pesquisador por um número reduzido de participantes nos estudos de representações sociais pode prejudicar a qualificação de "social" ao tipo de pensamento estudado. Isso, porque a construção do corpus da análise deve garantir a extensão do material textual em função dele representar os grupos considerados na análise. Afinal, só podemos falar de representações sociais no caso de saberes compartilhados, e uma indicação de que uma forma de compreensão de um dado objeto é efetivamente social envolve a recorrência de elementos e da estruturação entre eles nos membros identificados ou pertencentes a um grupo. ${ }^{19-20}$

No que diz respeito ao método de análise dos dados, os resumos da maioria das dissertações, $61(48 \%)$, e $10(26 \%)$ das teses não especificam o procedimento. A análise de conteúdo é o modo mais apontado em sete $(17,8 \%)$ dissertações e 46 $(36,8 \%)$ teses.

Os achados vêm ao encontro do que outros autores apontam, destacando ser uma prática comum de pesquisa em representações sociais a articulação da coleta de dados, através de entrevistas individuais com a técnica de tratamento, a análise de conteúdo, chamada como o "Romeu e Julieta" das representações. ${ }^{6}$

O uso de programas de computador software Alceste, EVOC e Tri-Deux-Mots são apontados em estudos a partir de 2002, usados individualmente ou em parceria com análise de conteúdo, detectados em $13(10 \%)$ das dissertações e seis $(15,7 \%)$ teses. Comumente os procedimentos de análise utilizam um software apropriado de modo complementar. O uso de computador pode indicar uma sofisticação dos procedimentos empregados, por meio de uso de recursos de organização e análise de dados. ${ }^{10}$
Os resultados aqui apresentados e discutidos mostram as diversas formas teóricas e metodológicas do uso da teoria das representações em dissertações e teses na área da enfermagem brasileira, o que revela também a apropriação ou não, pelos pesquisadores de área, sobre a teoria, suas possibilidades e aplicações.

\section{CONSIDERAÇÕES FINAIS}

Esse estudo possibilita responder ao objetivo de conhecer as escolhas teóricas e aspectos metodológicos da produção científica da enfermagem que envolve a Teoria das Representações Sociais, que constam no catálogo do CEPEn/ABEn. As pesquisas trazem contribuições reconhecidas, com temáticas envolvendo o processo de cuidar do ser humano, processo de trabalho, a dimensão educativa em enfermagem e saúde, entre outros. È possível identificar o crescente interesse dos pesquisadores pela teoria das representações sociais, demonstrando o reconhecimento da contribuição da teoria no desenvolvimento de estudos, considerando os aspectos psicossociais do processo saúdedoença na vida dos diversos grupos sociais.

O maior número de investigações é apontado nas dissertações de mestrado e evidencia um leque de opções metodológicas adotada nos estudos. A teoria das representações sociais possibilita o uso de diferentes métodos, qualitativos ou quantitativos para compreensão do seu objeto de pesquisa, assim como a adoção de diferentes perspectivas teóricas, como a estrutural, conhecida como teoria do núcleo central, e a processual, oriunda da teoria original de Moscovici.

É importante ressaltar a necessidade do desenvolvimento de outras investigações, com análises dos textos na íntegra, pois em muitos casos, os resumos apresentam lacunas em relação à descrição do percurso metodológico, os principais elementos da pesquisa e os autores não explicitam claramente o uso do referencial teórico.

Como se pode constatar, os resumos das dissertações e teses apresentam falta de informações e evidenciam a pouca importância ou desconhecimento dos autores sobre sua estrutura e finalidade. O resumo incluído nos catálogos tem o caráter informativo e permite divulgar e facilitar o acesso aos estudos produzidos na academia. Este deve constar do objetivo principal da investigação, assim como a metodologia, o referencial teórico, as técnicas de coleta ou produção de dados, os sujeitos, os métodos de tratamento dos dados, bem como os resultados e as conclusões. 
Como contribuição deste estudo, aponta-se a necessidade de maior critério na elaboração dos resumos, considerando que estes contêm as sínteses das pesquisas, que poderão despertar ou não o interesse no leitor, visto que os catálogos representam importante fonte de informações, e possibilitam a circulação e intercâmbio da produção científica construída em determinado campo do conhecimento.

\section{REFERÊNCIAS}

1. Moscovici S. A representação social da psicanálise. Rio de Janeiro: Zahar Editores; 1978.

2. Jodelet D. Representações sociais: um domínio em expansão. In: Jodelet D, organizadora. As representações sociais. Rio de Janeiro (RJ): UERJ; 2001.

3. Vala J, Monteiro MB, coordenadores. Psicologia Social $7^{\mathrm{a}}$ ed. Lisboa (PT): Fundação Calouste Gulbenkian; 2006.

4. Abric JC. A abordagem estrutural das representações sociais. In: Moreira ASP, Oliveira DC. Estudos interdisciplinares de representação social. $2^{\mathrm{a}} \mathrm{ed}$. Goiânia (GO): AB; 2000. p. 27-38.

5. Doise W. Cognições e representações sociais: a abordagem genética. In: Jodelet $\mathrm{D}$, organizador. As representações sociais. Rio de Janeiro (RJ): Ed. UERJ; 2001.

6. Sá CP. A construção do objeto de pesquisa em representações sociais. Rio de Janeiro (RJ): UERJ; 1998.

7. Guareschi P, Jovchelovitch S, organizadores. Textos em representações sociais. Petrópolis (RJ): Vozes; 1994.

8. Nascimento-Schulze,C. As representações sociais de pacientes portadores de câncer. In: Spink MJ, organizadora. São Paulo: Brasiliense; 1995. p.266-79.

9. Arruda A. Ecologia e desenvolvimento: representações sociais de especialistas em formação. In: Spink $\mathrm{M}$, organizador. O conhecimento cotidiano: As representações sociais na perspectiva da psicologia social. São Paulo: Brasiliense; 1995. p. 234-65.

10. Camargo BV, Wachelke JFR, Aguiar A. Desenvolvimento metodológico das pesquisas sobre representações sociais em jornadas internacionais de 1998 a 2005. In: Moreira ASP, Camargo B, organizadores. Contribuições para a teoria e o método de estudo das representações sociais. João Pessoa (PB): UFPB; 2007. p.181-202.

11. Silva AO, Alves MSCF, Moreira MASP, Silva SLF. Utilização da teoria das representações sociais no campo da saúde - UFPB - João Pessoa: Tendências e perspectivas. In: Coutinho MPL, Lima AS, Oliveira FB, Fortunato ML, organizadores. Representações sociais: abordagem interdisciplinar. João Pessoa (PB): Universitária/UFPB; 2003. p. 120-9.

12. Sá $\mathrm{CP}$, Arruda A. O estudo das representações sociais no Brasil. Rev Ciências Humanas. 2000; 3(esp):11-31.

13. Centro de Estudo de Pesquisa em Enfermagem, da Associação Brasileira de Enfermagem. Teses e Dissertações da ABEn-CEPEn [internet]. Brasília (DF): CEPEN; 2009 [acesso 2009 junho 19]. Disponível em: http:/ / enfermagem.bvs.br/php/index.php

14. Macias-Chapula CA. O papel da informetria e da cienciometria e sua perspectiva nacional e internacional. Ci Inf. 1998 Mai-Ago; 27(2):134-40.

15. Ravelli APX, Fernandes GCM, Barbosa SFF, Simão E, Santos SMA, Meirelles BHS. A produção do conhecimento em enfermagem e envelhecimento: estudo bibliométrico. Texto Contexto Enferm. 2009 Jul-Set; 18(3): 506-12.

16. Coordenação de Aperfeiçoamento de Pessoal de Nível Superior. Cursos recomendados [página na internet]. Brasília (DF): CAPES; 2011 [acesso 2011 abr 29]. Disponível em: http://www.capes.gov.br

17. Rogrigues RAP, Erdmann AL, Silva I, Fernandes JD, Araujo TL, Vianna LAC. Educação do doutorado em Enfermagem no Brasil. Rev Latino-am Enfermagem. 2008 Jul-Ago; 16(4):665-71.

18. Arruda A. Viver é muito perigoso: a pesquisa em representações sociais no meio do rodamoinho. In: Coutinho MPL, Lima AS, Oliveira FB, Fortunato ML, organizadores. Representações sociais: abordagem interdisciplinar. João Pessoa (PB): Universitária/ UFPB; 2003. p.11-31.

19. Nascimento-Schulze, C, Camargo BV. Psicologia social, representações sociais e métodos.Temas Psicol. 2000 Dez; 8(3):287-99.

20. Ferreira NSA. As pesquisas denominadas estado da arte. Educ Soci. 2002 Ago; 23(79):257-72. 